\title{
Evaluación del Programa de Servicio Social UADY-Peraj “Adopta un amig@" de la Universidad Autónoma de Yucatán.
}

\author{
Evaluation of the UADY-Peraj Social Service Program "Adopta un amig@" \\ from the Autonomous University of Yucatan
}

David de Jesús Santoyo Manzanilla

\section{RESUMEN}

Este trabajo se realizó con el propósito de investigar el impacto que la aplicación del Programa de Servicio Social UADY-Peraj "Adopta un Amig@" ejerce sobre los niños en riesgo social que participan en él y, de esta manera, apoyar el desarrollo social de estos alumnos de educación primaria de escuelas públicas, a través de la tutoría de jóvenes universitarios prestadores de servicio social.

El estudio se llevó a cabo mediante un enfoque positivista de tipo cuantitativo y se administraron cinco cuestionarios a los participantes para recolectar información. Los resultados que arrojaron estos instrumentos permitieron conocer el grado de progreso de los niños participantes en riesgo social en los ámbitos escolar, personal, familiar y social.

\section{PALABRAS CLAVE:}

Adaptación social, bienestar social, cambio de actitudes, cambio educacional, detección de necesidades, programas sociales, tutoría, servicio social.

\section{ABSTRACT}

This work was carried out to know the impact that the UADY-Peraj Social Service Program "Adopts a Friend" has on the children at social risk that participate in it and, as well to support the social development of these students of primary education of public schools, through the tutoring of young university providers of social service.

The study was carried out using a positive quantitative approach and five questionnaires were given to the participants to gather information. The results of these instruments revealed the degree of progress of children participating in social risk in the next dimensions: school, personal, family and social settings.

\section{KEYWORDS}

Social adaptation, social welfare, change of attitudes, educational change, detection of needs, social programs, tutoring, social service.

\section{EL PROYECTO PERAJ}

Peraj significa flor en el idioma hebreo, pero son también las siglas de un relevante proyecto de tutorías para niños y adolescentes iniciado en Israel en 1974 bajo los auspicios del Instituto Weizmann de Ciencias, uno de los institutos líderes del mundo en investigación multidisciplinaria en dicho país. Dentro del marco de este programa, estudiantes de todas las universidades del país enseñan y educan a alumnos en riesgo social, quienes necesitan apoyo y guía personal en sus estudios. Es el principal programa tutorial de Israel, aproximadamente el $15 \%$ de los estudiantes de licenciatura de toda la nación israelita participa en él. Actualmente Peraj apoya el desarrollo de proyectos similares en más de 20 países alrededor del mundo (Peraj-México 2013).

Entre 1974 y 2004 el programa se implementó en 15 países alrededor del mundo. Para el 2007 se forma la alianza entre la Secretaría de Educación Pública (SEP), el Programa Nacional de Becas para la Educación Superior (PRONABES) y la Asociación Nacional de Universidades e Instituciones de Educación Superior (ANUIES), integrando a 12 universidades públicas y a una privada, la Universidad de las Américas de Puebla (Vilchis, 2012).

La experiencia más cercana a México es el Programa "Adopta un Hermano" que se organiza en Chile desde 2001. En ambos países, el modelo de tutorías ha logrado en los menores la disminución de las tasas de deserción escolar y de participación en actividades delictivas, favoreciendo un desarrollo positivo de los niños en su ámbito escolar, personal, familiar y social (UNAM-Peraj, 2014). 
Perspectivas Docentes 64

Evaluación del Programa de Servicio Social UADY-Peraj “Adopta un amig@” de la Universidad Autónoma de Yucatán.

Evaluation of the UADY-Peraj Social Service Program "Adopta un amig@" from the Autonomous University of Yucatan

David de Jesús Santoyo Manzanilla

En México, PERAJ-México es una asociación civil sin fines de lucro que es la responsable del control, desarrollo y mantenimiento del programa Peraj-Adopta un amig@. A semejanza de otros países, este programa de servicio social tutorial a nivel nacional apoya el desarrollo social de alumnos de educación primaria de escuelas públicas (los amig@s), a través de la tutoría de jóvenes universitarios prestadores de servicio social (los tutores) buscando que, por medio de una relación significativa entre tutor y amig@, se apoye el desarrollo educativo, social y psicológico del menor.

Los tutores apoyan a los menores con el propósito de fortalecer su autoestima, desarrollar sus habilidades sociales, mejorar sus hábitos de estudio y ampliar su cultura general y sus horizontes. Los tutores se convierten en un modelo positivo que seguir para el niño de tal modo que se cumpla el pensamiento bíblico que inspira el programa: "Instruye al niño en su camino, y aun cuando fuere viejo no se apartará de él" (Proverbios 22,6) (Peraj-México, 2013).

El Programa PERAJ fue presentado por primera vez en México en la Universidad Nacional Autónoma de México por la Asociación de Amigos del Instituto Weizman de Ciencias para lo cual se retomó la filosofía del programa PERAJ israelí adecuándolo al contexto mexicano, enfatizando dos estrategias fundamentales: la atención a niños de escuelas públicas y la participación de jóvenes universitarios.

A partir de mayo del 2003 se puso en operación el Programa de Servicio Social Tutorial UNAM-Peraj "Adopta un amig@", siendo la primera universidad mexicana en llevar a cabo el programa en el cual jóvenes universitarios fungen como tutores de niños entre 8 y 12 años de edad. En el año 2009, en el estado de Michoacán, se llevó a cabo un taller de seguimiento del programa PERAJ-Adopta un amig@ en el que participaron 24 universidades de distintas partes del país. En dicho encuentro se trataron los temas referentes a la importancia de la labor que los universitarios realizan en su servicio social en el cual fungen como tutores de los niños; se concluyó que estas tutorías benefician el desarrollo social, psicológico y educativo de los menores que participan en él (ITSON, 2009).

En la región sureste de México fue la Universidad Autónoma de Yucatán la que inicia este programa en enero de 2008 con una duración de únicamente seis meses por tratarse del pilotaje del programa. A partir de septiembre de ese mismo año se comienza el programa con una duración de 10 meses de manera ininterrumpida hasta la fecha.
En Peraj, la formación integral del niño se considera como "un estilo educativo que pretende no sólo instruir a los estudiantes con los saberes específicos de las ciencias sino, también, ofrecerles los elementos necesarios para que crezcan como personas buscando desarrollar todas sus características, condiciones y potencialidades" (ACODESI, 2003).

Desde sus orígenes, este programa ha buscado primordialmente, beneficiar a los niños en riesgo atendiendo seis dimensiones de la personalidad: afectiva, social, cultural, escolar, motivacional y comunicativa. El programa enuncia que no sólo los amigos son los favorecidos sino también los tutores, padres y maestros de los niños participantes, puesto que al elevar la calidad de las actitudes o respuestas de estos niños frente a sus retos cotidianos, también las personas que pertenecen a su círculo de relaciones se verán afectadas de manera positiva (Lineamientos Peraj, 2011).

\section{EL PROGRAMA PERAJ-ADOPTA UN AMIG@: SUS BENEFICIOS}

Con el fin de abatir los altos índices de deserción y rezago atribuidos a diversos factores, como son la falta de interés de parte de los alumnos, ya sea por problemas socioeconómicos o personales; la insuficiente atención de las instituciones educativas en la formación integral del estudiante, así como la escasa participación de los docentes en la búsqueda de soluciones para estos problemas, se han implementado estrategias y acciones que favorecen en los estudiantes el desarrollo de habilidades y actitudes no considerados dentro del plan de estudios. Con ello se pretende no sólo resolver el problema de rezago y deserción, sino elevar el índice de eficiencia terminal, así como la calidad de la educación.

Para lograr sus objetivos se enfatiza la importancia del tutor en el programa UADY-Peraj Adopta un amig@ para apoyar a niños que se encuentran en situación de riesgo social; se describe el impacto de los apoyos educativo, afectivoformativo y social que reciben los niños en riesgo social que participan en el programa así como las áreas de formación integral relacionadas con el programa y los niños, quienes de alguna u otra manera sufren cambios en su desarrollo social, psicológico y educativo.

El Programa "Adopta un amig@" es un programa de servicio social que vincula a jóvenes universitarios que fungen como tutores de niños de entre 8 y 12 años de edad, de escuelas 
Perspectivas Docentes 64

Evaluación del Programa de Servicio Social UADY-Peraj “Adopta un amig@” de la Universidad Autónoma de Yucatán.

Evaluation of the UADY-Peraj Social Service Program "Adopta un amig@" from the Autonomous University of Yucatan

David de Jesús Santoyo Manzanilla

públicas cercanas a los planteles universitarios, durante un ciclo escolar. Al tiempo de establecer una relación significativa, el programa se propone fortalecer:

- El desarrollo social, psicológico y educativo del menor mediante estrategias pedagógicas, y

- La formación profesional y humana del joven universitario, y la realización de su compromiso de retribución social, a través de la organización de actividades lúdico-recreativas.

- La tutoría, entendida como una labor docente por medio de la cual un profesor brinda orientación y acompañamiento a sus alumnos considerando sus necesidades educativas individuales, con el propósito de facilitar su aprendizaje escolar y promover su desarrollo integral, representa una de las acciones implementadas con resultados bastante alentadores.

Entre los objetivos del Programa "Adopta un amig@", se encuentran apoyar el desarrollo de los menores en las áreas siguientes:

- Escolar-educativa. Mejorando su percepción y motivación con respecto a las actividades escolares y fortaleciendo los hábitos de estudio.

- Afectiva-formativa. Fortaleciendo la autoestima de los niños, facilitando la expresión de su afectividad y posibilitando su capacidad para establecer vínculos interpersonales.

- Social. Desarrollando sus habilidades sociales y creándoles un sentido de pertenencia, integración y compromiso social.

- Lúdico-cultural. Realizando visitas y actividades que permitan a los niños abrir su visión y enriquecer su bagaje cultural, científico y recreativo (UNAM-Peraj, 2014).

En lo que respecta a los estudiantes universitarios que realizan su servicio social, en calidad de tutores, los beneficios que les reporta el programa "Adopta un amig@", se encuentran:
- Favorecer su formación integral y compromiso social mediante la atención a la población vulnerable y en riesgo.

- Desarrollar su capacidad como facilitadores del aprendizaje y generadores de vínculos socio-afectivos para el crecimiento personal, y el logro de metas y objetivos.

- Fortalecer sus habilidades complementarias al desempeño profesional tales como creatividad, iniciativa, trabajo en equipo, expresión oral y escrita, organización de eventos, autorregulación e integración.

- Consolidar la aplicación de los conocimientos disciplinarios adquiridos mediante la identificación, diseño, elaboración e impartición de temáticas útiles para la formación de los niños, de manera dinámica y atractiva que favorezca el interés permanente por todo tipo de conocimiento (UNAM-Peraj, 2014).

\section{LA TUTORÍA COMO ESTRATEGIA RECTORA}

Según Argüis (2005), la tutoría es una actividad inherente a la función del profesor, que se realiza individual y colectivamente con los alumnos de un grupo-clase, con el fin de facilitar la integración personal en los procesos de aprendizaje. Por tal motivo, se puede señalar que la tutoría y acción tutorial son dos conceptos complementarios y significan el conjunto de las actuaciones de orientación personal, académica y profesional diseñado por los profesores con la colaboración de los alumnos y de la misma institución.

Menchén (2003), plantea que el tutor es la persona que, a través de la enseñanza, ayuda al alumno o alumna, a optimizar sus capacidades y formar su personalidad, mediante el descubrimiento de la realidad y la cultura, de tal forma que pueda vivir satisfactoriamente.

Mora (2009), señala que, la tutoría es un encuentro, pretexto para el dialogo, donde el encuentro con otro ser humano en desarrollo, se vuelva factor de intercambios humanos que enriquezcan, la subjetividad de uno y otro en un intercambio sin fin. Así entendida, la importancia tutorial puede ser un factor de prevención, que resuelva algunas de las dificultades en que se ve entrampado un joven descuidado 0 abandonado que se caracteriza por un desdén de los lazos afectivos de los 
Perspectivas Docentes 64

Evaluación del Programa de Servicio Social UADY-Peraj “Adopta un amig@” de la Universidad Autónoma de Yucatán.

Evaluation of the UADY-Peraj Social Service Program "Adopta un amig@" from the Autonomous University of Yucatan

David de Jesús Santoyo Manzanilla

cuales no se espera nada, más allá de lo que es necesario para sobrevivir.

El tutor es un hombre que, en su labor de educador, se ha de relacionar con muchas personas mediante el trato continuo con los alumnos, con los padres, profesores, directivos, etc. Esto exige realmente mucho, ya que es el responsable de crear un clima de comunicación de relación amistosa y de colaboración.

En este sentido, el Programa "Adopta un amig@”, señala que las funciones del tutor de los estudiantes universitarios que realizan su servicio social son:

1. Crear un clima de confianza que propicie el conocimiento de los distintos aspectos que puedan influir directa 0 indirectamente en el desempeño escolar de los niños.

2. Conocer las expectativas, intereses y limitaciones que tiene el niño.

3. Fomentar en el niño una actitud perseverante y de logro, como una forma de evidenciar y fortalecer sus capacidades.

4. Facilitar las condiciones para que el niño plantee sus dudas.

5. Explorar y promover las competencias y capacidades de estudio en el niño.

6. Generar las condiciones para que los niños adquieran las herramientas que les permitan recrear el conocimiento y prepararse para el siguiente nivel educativo.

7. Identificar los problemas académicos y personales que afectan el desarrollo escolar del niño, con la finalidad de canalizarlo cuando requiera una atención especializada.

8. Motivar al niño para que participe y se integre a las actividades del grupo.

9. Promover el análisis y la reflexión con los niños, como un instrumento de enseñanza-aprendizaje.
10. Socializar, discutir y consensuar entre el equipo de tutores, los programas y actividades que fortalezcan su papel, así como las competencias convenientes para el tipo de tutoría que brindará.

11. Ayudar al fortalecimiento de las habilidades expresivas y de comunicación de los amig@s participantes (UNAM-Peraj, 2014).

\section{EVALUAR PARA ASEGURAR LA INCIDENCIA}

De acuerdo con los Lineamientos del Programa Peraj (2011) se recomienda llevar a cabo una evaluación sobre amig@s y tutores mediante la aplicación de instrumentos de evaluación al inicio y al final del programa a fin de medir los cambios y el impacto logrado por el programa en las áreas en las que éste incide: afectiva, social, cultural, escolares, motivacional y de comunicación.

El proyecto de evaluación de este programa pretende conocer si los participantes/tutorados han presentado avances 0 progresos en cuanto a su formación integral al formar parte del proyecto "Adopta un Amig@", así como verificar el cumplimiento de los objetivos establecidos en el proyecto. Es decir, la evaluación está centrada en los niños o amigos que participan en el programa y que presentan alguna situación de riesgo social o académico.

Además, desde el punto de vista social e institucional, estos estudios podrán dejar ver la contribución del programa para los niños y permitirán a los responsables del programa contribuir, junto a las otras instituciones de educación superior (IES), a un mejor desarrollo y consolidación del Programa Peraj. Por ello se considera que es esencial medir la importancia de la aplicación de este programa de servicio social para niños en riesgo social.

\section{METODOLOGÍA}

La investigación es de tipo cuasi experimental con un diseño de dos grupos. El objetivo primordial por parte del investigador es detectar los efectos del programa UADY-Peraj "Adopta un Amig@" en los sujetos participantes.

Cook (1983), citado por Hernández, Fernández y Baptista (2010), define la investigación cuasi-experimental como una 
Perspectivas Docentes 64

Evaluación del Programa de Servicio Social UADY-Peraj “Adopta un amig@” de la Universidad Autónoma de Yucatán.

Evaluation of the UADY-Peraj Social Service Program "Adopta un amig@" from the Autonomous University of Yucatan

David de Jesús Santoyo Manzanilla

clase de estudios que se llevan a cabo fuera del laboratorio y no implican asignación aleatoria de las unidades experimentales a las condiciones de tratamiento. Es cuasi experimental ya que los sujetos que participan en el programa no se seleccionan al azar sino que se toman en cuenta criterios de inclusión.

El diseño se llevó a cabo con prueba-posprueba de tipo secuencial a grupos a los que se les administró una prueba al inicio. Dicha prueba sirvió para verificar la equivalencia inicial de los grupos (niños en riesgo social) y después de un tiempo se aplicó la posprueba a fin de analizar los efectos de la administración del tratamiento.

Para la determinación de la muestra de esta investigación se tomaron en cuenta los siguientes criterios para la formación de los grupos de tratamiento:

- El niño en riesgo es recomendado por otro amigo.

- El niño se encuentra en quinto y sexto de primaria y no ha participado anteriormente en el programa.

- El niño en riesgo será elegido por la zona en la que vive y mediante la interacción durante el programa tanto con el niño como los padres y amigos.

La población en la presente investigación la conforman en total 34 alumnos en riesgo social, de los cuales 17 alumnos provienen de la Escuela Primaria Agustín Vadillo Cícero ubicada en la colonia San Antonio Xluch III de la ciudad de Mérida y 17 de la Escuela Primaria Centenario del Ejército Mexicano localizada en la colonia Libertad II. La muestra está constituida por un total de 21 alumnos en riesgo social quienes cumplen con los criterios de inclusión mencionados anteriormente.

Cabe destacar que la elección de dichas instituciones se realizó tomando en cuenta la declaración de la SEP, en cuanto a que estén consideradas como escuelas de calidad, aunque no se establece como criterio de selección. De igual manera, fueron escogidas por la cercanía al entorno inmediato del plantel educativo en donde se llevó a cabo el programa, con el fin de facilitar la asistencia de los niños. En este caso las sedes fueron la Ex Facultad de Ingeniería Química y la Unidad Académica Bachillerato con Interacción Comunitaria (UABIC), ambas de la Universidad Autónoma de Yucatán (UADY).

\section{EVALUACIÓN CON BASE A INDICADORES}

La evaluación del impacto del desempeño del programa en los niños 0 amig@s se basa en indicadores que muestran el progreso del niño en las áreas afectiva, social, cultural, escolar, motivacional y comunicativa. Para llevar a cabo esta medición, PERAJ-México diseñó una serie de instrumentos que las instituciones participantes deberán aplicar para dar cuenta del impacto del programa en los participantes, sobre todo, en su entorno escolar, familiar y social.

Tales instrumentos se desarrollaron a partir de los resultados de las experiencias y recomendaciones de la aplicación del programa Peraj-Adopta un Amig@ en las primeras trece instituciones de educación superior que participaron en este programa en México.

La primera prueba es una de autoestima llamada AUCASO proporcionada por la Dirección General de Orientación y Servicios Educativos (DGOSE) de la Universidad Nacional Autónoma de México (UNAM) y se trata de un instrumento estandarizado para medir la autoestima. La segunda prueba que se aplicó fue el cuestionario "Sobre mí mismo" que mide las seis áreas de desarrollo indicadas en el programa PerajAdopta un Amig@ que fueron mencionadas con anterioridad. La tercera prueba se llama "Tutor Peraj sobre amig@” y mide los cambios en las dimensiones de desarrollo contempladas por el programa desde la perspectiva del tutor. La cuarta prueba se llama "Maestro sobre el alumn@" y mide los cambios en las áreas de desarrollo contempladas por el programa desde la perspectiva del profesor de su escuela primaria. El quinto cuestionario se titula "Padre de familia o responsable sobre el amig@” y mide la percepción del padre de familia o tutor con respecto a las áreas de desarrollo contempladas por el programa.

\section{RESULTADOS}

Para entrar en contexto, en la sede de la Ex FIQ se contó con una muestra de nueve sujetos, seis hombres $(66.7 \%)$ y tres mujeres $(33.3 \%)$, con un rango de edad de nueve y once años mientras que en la cede UABIC se contó con una muestra de doce sujetos, ocho hombres (66.7\%) y cuatro mujeres (33.3\%), con un rango de edad de nueve y trece años. 
Perspectivas Docentes 64

Evaluación del Programa de Servicio Social UADY-Peraj “Adopta un amig@” de la Universidad Autónoma de Yucatán.

Evaluation of the UADY-Peraj Social Service Program "Adopta un amig@" from the Autonomous University of Yucatan

David de Jesús Santoyo Manzanilla

Para responder a los objetivos de la investigación, se presenta el promedio de los puntajes obtenidos en las pruebas en una escala de 100 puntos, organizado por sede y tiempo de prueba, tal como se describe a continuación.

\section{Gráfica 1. Prome dio de puntajes obtenidos en las fases de prueba y} pos prueba en la sede Ex FIQ

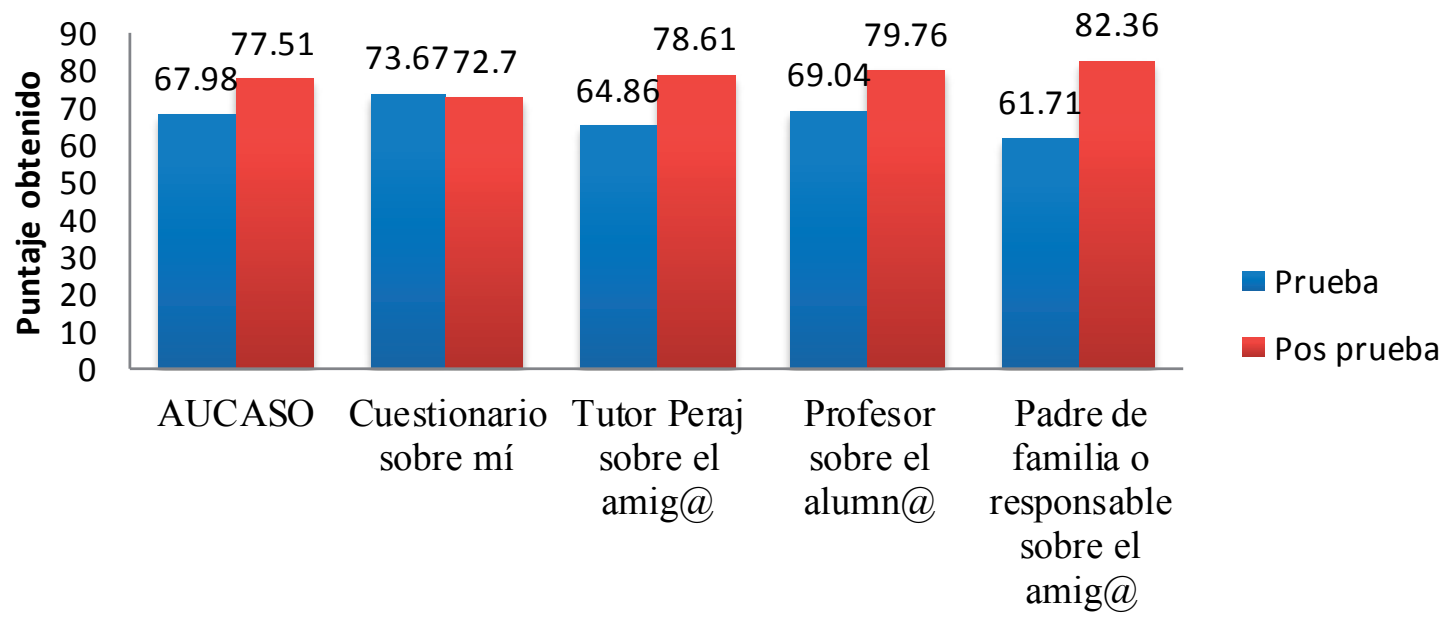

Como puede observarse, la Gráfica 1 corresponde al promedio de los puntajes obtenidos en cada una de las pruebas administradas a los niños participes de la sede Ex FIQ para ambas fases. Se puede analizar que el instrumento "Cuestionario sobre mí mismo" el valor de la pos prueba descendió en 97 puntos en comparación de la fase de prueba, mientras que el resto de los instrumentos señala una mejoría en la fase de pos prueba con respecto a la de prueba.

Gráfica 2. Prome dio de puntajes obtenidos en las fases de prue ba y pos prue ba en la sede UABIC

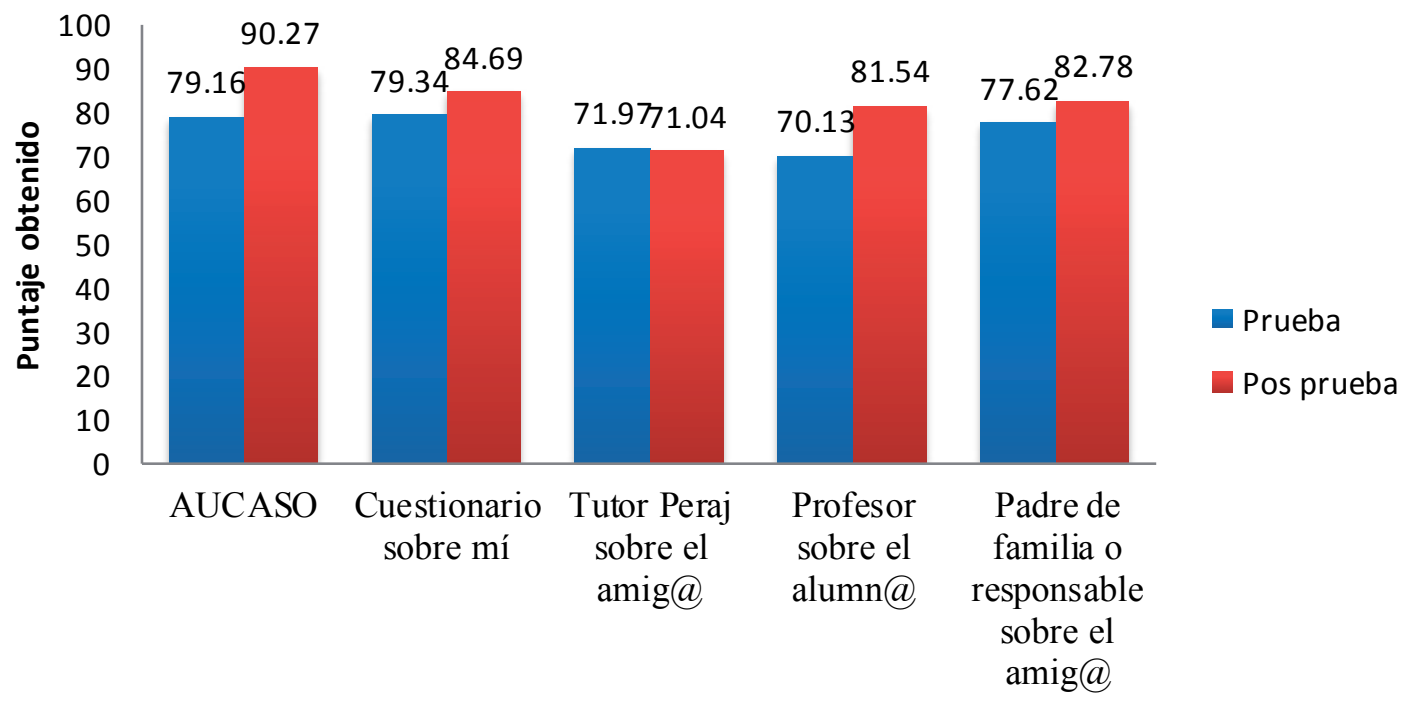


Perspectivas Docentes 64

Evaluación del Programa de Servicio Social UADY-Peraj “Adopta un amig@” de la Universidad Autónoma de Yucatán.

Evaluation of the UADY-Peraj Social Service Program "Adopta un amig@" from the Autonomous University of Yucatan

David de Jesús Santoyo Manzanilla

En la Gráfica 2, se observan los promedios de los puntajes obtenidos de las pruebas administradas en la sede UABIC, de igual manera, se analiza que la fase de pos prueba señala una mejoría con respecto a la fase de prueba, salvo en el instrumento "Tutor Peraj sobre el amig@", el cual reporta un descenso de .93 puntos en la fase de pos prueba con respecto a la de prueba.

\section{DISCUSIÓN}

Esta investigación tuvo el propósito de investigar el impacto que la aplicación del Programa de Servicio Social UADY-Peraj "Adopta un Amig@" ejerce sobre los niños en riesgo social que participan en él. Sobre todo, se pretendió apoyar el desarrollo social de estos alumnos de educación primaria de escuelas públicas mediante la identificación de las áreas de oportunidad que prevalecen a pesar de tener un desarrollo favorable en el Programa, los cuales se manifiestan en sus diferentes dimensiones la personalidad: afectiva, social, cultural, escolar, motivacional y comunicativa.

De los resultados obtenidos en esta investigación, se puede deducir que el programa de servicio social, tiene un efecto positivo en el desarrollo social de los alumnos que participan en él independientemente de la sede en la que se imparte, ya que ambas reportan una mejoría en la fase de pos prueba en cuatro instrumentos de los cinco administrados; sin embargo, las diferencias observadas en los puntajes obtenidos no son las esperadas, ya que se existen diferencias que van desde los -.9 hasta los 17 puntos, ninguna rebasa los 20 puntos de diferencia.

Estos resultados pueden deberse al tiempo de duración del programa "Adopta un amig@", pues no se puede afirmar que el tiempo designado para su implementación logre formar un vínculo estrecho que permita una correcta influencia positiva del tutor al tutorado. Otro factor que pudo influir, es el perfil de cada tutor, pues se reportan diversos perfiles no vinculados al área socio-formativa o educación y humanidades, como lo son licenciados en educación de las matemáticas, contadores, arquitectos, ingenieros físicos y biólogos.

A pesar de haber una mejoría de las dimensiones, prevalecen algunas áreas de oportunidad después de concluir el programa, como lo son: la autoimagen negativa, la toma de decisiones, la educación autodidacta, y la expresión de ideas. Cabiendo aclarar que se reportó una mejoría de estas, pero no de manera elevada.

Estos resultados coinciden con otras investigaciones realizadas como la de Martínez y Hernández realizada en 2012, cuya investigación determinó que el impacto del programa PerajAdopta un amig@ es favorable para los niños que participan en el programa, esto llevado a cabo a través de instrumentos similares a los utilizados para esta investigación. Aclarando que la diferencia de ambas investigaciones, radica en que no se contempla para este estudio, la inclusión del impacto del programa en los tutores, pues se considera como un hecho más complejo que debe ser abordado desde un enfoque más holista.

Gracias a estos resultados se reafirman las ideas sobre los beneficios de contar con un sistema de tutorías como sistema de aprendizaje individualizado por la relación alumno tutor, pues se reconocen las necesidades y se establecen planes que respondan a los problemas y necesidades de los tutorados (Medina, 2011). A su vez, la orientación educativa, la cual desde una perspectiva del desarrollo, se considera como un proceso evolutivo con tendencia a la prevención, que favorece el desarrollo de todos los participantes (Ortiz, 2011). Pero sobre todo, asegura que la función tutorial cumple con su propósito de resolver los problemas que puedan afectar al desarrollo intelectual y emocional de tutorado pues la tutoría auxilia al alumno en situaciones relacionadas con la integración con la comunidad, deficiencias académicas, bajo rendimiento, cambios psicológicos, baja autoestima, conducta de riesgo (Carcaño, s.f.).

Cabe aclarar que la fiabilidad y validez de la investigación surge de la participación de los sujetos y su compromiso para iniciar y finalizar el programa "adopta un amig@", pues por experiencia de implementaciones anteriores se sabe que existe la deserción del programa tanto por los tutores como de los tutorados, así como al uso de los instrumentos recomendados y facilitados por la Universidad Nacional Autónoma de México elaborados para este programa, los lineamientos pertinentes del programa "Adopta un amig@" y los pilotajes y ajustes realizados para el contexto socioeconómico en el cual se realizó la investigación.

\section{CONCLUSIONES}

La creación de una evaluación sobre el grado de desarrollo alcanzado al concluir el programa de servicio social, permite 
Perspectivas Docentes 64

Evaluación del Programa de Servicio Social UADY-Peraj “Adopta un amig@” de la Universidad Autónoma de Yucatán.

Evaluation of the UADY-Peraj Social Service Program "Adopta un amig@" from the Autonomous University of Yucatan

David de Jesús Santoyo Manzanilla

conocer los aspectos a mejorar en el programa, en todas las fases de su implementación como lo son: la selección de los tutores, las actividades desarrolladas, el tiempo de implementación y la definición de metas del programa.

En cuanto a la metodología empleada para el desarrollo de esta investigación, se puede decir que el diseño de dos fases permite una visión de los panoramas al inicio y termino del programa, lo que permite identificar las dimensiones que tuvieron un cambio favorable y cuales permanecen como áreas de oportunidad, logrando de esta manera identificar si existió un desarrollo significativo.

Existe un desarrollo favorable en cuatro de los instrumentos administrados, mientras que no existe una diferencia significativa por parte del quinto instrumento para ambas sedes, lo que nos conlleva a decir que el programa de servicio social "adopta un amig@" cumple con su objetivo de apoyar el desarrollo social de estos.

Así, se enlista a continuación una serie de sugerencias generales de la investigación:

- Debido a que es la primera investigación que se realiza con respecto a la implementación del programa de servicio social "adopta un amig@" en este contexto se debe trabajar en la promoción de estos resultados para alentar a futuras investigaciones en beneficio del programa, tanto para contextos nacionales e internacionales.

- Es importante establecer una metodología claramente definida para la administración de los instrumentos, pues ambas fases dependen de medir las actitudes iniciales (sin influencia alguna del programa) y finales (al concluir el programa).

- Las futuras investigaciones relacionadas con este programa de servicio social, deben diferir en objetivos y no reproducir esta investigación con nuevos participantes, para lograr un panorama holista que comprenda otras dimensiones y variables.

- Los instrumentos facilitados por la UNAM, deben ser reorganizados por las dimensiones que miden cada reactivo, así como cambiar la redacción negativa a positiva que existe en varios instrumentos, pues dificulta la forma de respuesta y el análisis de los resultados.

- Incluir un estudio sobre como el programa afecto a los tutores (enfoque cualitativo, para tener una visión holista).

- Sugerencias específicas con base en los resultados:

- Implementar un sistema de evaluación continua sobre el desarrollo alcanzado por los alumnos al finalizar el ciclo del programa.

- Crear un seguimiento de los alumnos, con la finalidad de lograr hacerlos participes en un nuevo ciclo del programa.

- Estudiar con mayor detalle cada una de las dimensiones que componen el desarrollo integral del alumno, con la finalidad de investigarlas a profundidad por separado.

- Establecer un perfil único para los tutores participantes, que considere su formación y sus habilidades para tutoría.

- Crear conciencia sobre la participación de todos los actores, ya que se tiene información de que existe deserción del programa por ambas partes. 
Perspectivas Docentes 64

Evaluación del Programa de Servicio Social UADY-Peraj “Adopta un amig@” de la Universidad Autónoma de Yucatán.

Evaluation of the UADY-Peraj Social Service Program "Adopta un amig@" from the Autonomous University of Yucatan

David de Jesús Santoyo Manzanilla

\section{REFERENCIAS BIBLIOGRÁFICAS}

Argüis, R., et al. (2005). La Acción Tutorial. Barcelona. Ed. Grao.

Asociación de Colegios Jesuitas de Colombia, ACODESI. (2003). La Formación Integral y sus Dimensiones: Texto Didáctico. Recuperado de http://www.acodesi.org.co/es/ images/Publicaciones/pdf libros/texto\%20did\%E1ctico\%20 -\%20negro.pdf

Carcaño, M. (s.f.). Ponencia: Reflexión sobre la función tutorial. Recuperado de https://es.slideshare.net/HumanidadesUCSF/ reflexion-28023022

Cook, D. (1983). Quasi-experimentation: Its ontology, epistemology and methodology. In Beyond Method: Strategies for Social Research. Beverly Hills: Sage.

Hernández S, Fernández C., y Baptista L. (2010). Metodología de la Investigación. Cuarta Edición. México: McGraw-Hill.

ITSON y su gente. Instituto Tecnológico de Sonora. Julio de 2009, No. 541. Recuperado de: http://www.itson.mx/ publicaciones/itsonysugente/Documents/itsonysugente541.pdf

Martínez, L. y Hernández, L. (2012). Impacto del programa Peraj en el amig@: La tutoría como estrategia de fortalecimiento de los recursos psicológicos. Facultad de Psicología. Universidad Michoacana de San Nicolás de Hidalgo. Recuperado de http://bibliotecavirtual.dgb.umich. mx:8083/jspui/bitstream/123456789/6610/1/Impacto $\% 20$ del\%20programa\%20Peraj\%20en\%20el\%20amig@.\%20 La\%20tutor\%C3\%ADa\%20como\%20estrategia\%20de $\% 20$ fortalecimiento $\% 20$ de $\% 2010$ s $\% 20$ recursos $\% 20$ psicol\%C3\%B3gicos.pdf
Medina, E. (2011). La importancia de la tutoría en los procesos educativos. Recuperado de www.oem.com.mx/elsoldedurango/ notas $/ \mathrm{n} 2341026 . \mathrm{htm}$

Menchen, F. (2003). El Tutor. Madrid. Editorial CCS.

Mora, F. (2009). Tutoría en la Educación. México. Ed. Fomento Científico Monterey.

Ortiz, M. (2011). Ponencia: Importancia de la orientación educativa y tutoría en algunos niveles de intervención. Recuperado de: http://ujed.mx/Todo/documentos/pdf/ eje_2/2_09.pdf

Peraj-México. (2011). Adopta un amig@. Lineamientos Peraj.

UNAM-PERAJ. Programa de servicio social tutorial UNAM PERAJ “ADOPTA UN AMIG@”. México. Recuperado de: http:/l www.dgoserver.unam.mx/portaldgose/servicio-social/archivos/ ejemplos/UNAMPerajAdoptaunamigo.pdf.

Vilchis, D. (2012). Análisis Sociológico del Programa UPN-Peraj Adopta a un Amigo. México, D.F.: Universidad Pedagógica Nacional. 